\title{
What do we know about the proton spin structure? ${ }^{1}$
}

\author{
Mauro Anselmino \\ Dipartimento di Fisica Teorica, Università di Torino and \\ INFN, Sezione di Torino, Via P. Giuria 1, I-10125 Torino, Italy
}

\begin{abstract}
A brief summary of the theoretical and experimental knowledge of the spin structure of the proton is presented. The helicity distributions of quark and gluons are discussed, together with their related sum rules. The transversity distribution is also introduced with possible strategies for its measurement. Novel spin dependent and $\boldsymbol{k}_{\perp}$ unintegrated distribution and fragmentation functions are discussed, in connection with a new and rich phenomenology of transverse single spin asymmetries.
\end{abstract}

\section{Introduction}

The spin nucleon structure - as observed in high energy, short distance interactions - is schematically described in Fig. 1.

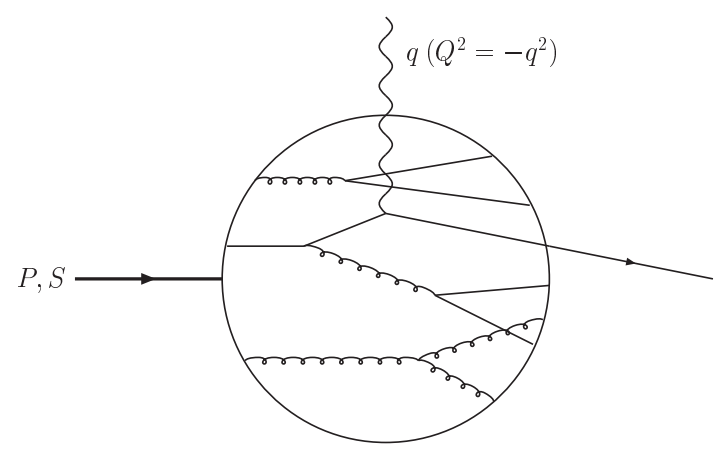

Figure 1: The nucleon structure, as seen by a high energy, large $Q^{2}$, probe. The nucleon breaks into unobserved final particles $X$; the ejected parton may fragment into an observed hadron.

The large $Q^{2}$ probe - typically, a virtual photon - "sees" QCD partons, carrying a longitudinal momentum fraction $x$, and their interactions, with gluon and

\footnotetext{
${ }^{1}$ Talk delivered at the 2nd International Symposium on the Gerasimov-Drell-Hearn sum rule and the spin structure of the nucleon, GDH 2002, July 3-6 2002, Genova, Italy
} 
$q \bar{q}$ pair creation; the information about such a complicated structure is usually collected via measurements of the Deep Inelastic Scattering (DIS) cross-section and storaged in the structure functions which appear in the most general expression of the cross-section. When neglecting weak, parity violating contributions, there are 2 unpolarized $\left(F_{1}, F_{2}\right)$ and 2 polarized $\left(g_{1}, g_{2}\right)$ structure functions: perturbative QCD allows a simple partonic interpretation of $F_{2}$ and $g_{1}\left(F_{1}\right.$ is related to $F_{2}$ while $g_{2}$ does not have a partonic interpretation). We only consider here the polarized proton structure trying to summarize in a short time and space the main ideas, the most recent results and the open problems; many detailed and comprehensive reviews on the subject can be found in the literature [1].

The main issues and questions we are going to discuss here are:

- our knowledge about the polarized structure functions $g_{1}\left(x, Q^{2}\right)$ and $g_{2}\left(x, Q^{2}\right)$ and about quark and gluon helicity distributions, $\Delta q\left(x, Q^{2}\right)$ and $\Delta g\left(x, Q^{2}\right)$; how well do we know them?

- are fundamental sum rules satisfied and what do we know about quark and gluon orbital angular momentum, $L_{q}$ and $L_{g}$ ?

- $\Delta q\left(x, Q^{2}\right), \Delta g\left(x, Q^{2}\right), L_{q}$ and $L_{g}$ are not the whole story: how and where do we learn about the transversity distribution $h_{1}\left(x, Q^{2}\right)$ ?

- could we learn more and understand more from intrinsic $\boldsymbol{k}_{\perp}$ unintegrated distribution and fragmentation functions?

\section{The longitudinally polarized proton and the helicity distributions}

At NLO in the QCD parton model the structure function $g_{1}$ is given by

$$
g_{1}\left(x, Q^{2}\right)=\frac{1}{2} \sum_{q} e_{q}^{2}\left\{\Delta C_{q} \otimes[\Delta q+\Delta \bar{q}]+\frac{1}{N_{f}} \Delta C_{g} \otimes \Delta g\right\}
$$

where $\Delta q\left(x, Q^{2}\right)$ and $\Delta g\left(x, Q^{2}\right)$ are respectively the quark (of flavour $q$ ) and gluon helicity distributions; we have, as usual, defined the convolution

$$
\Delta C \otimes \Delta q \equiv \int_{x}^{1} \frac{d y}{y} \Delta C\left(\frac{x}{y}, \alpha_{s}\right) \Delta q\left(y, Q^{2}\right)
$$

and the coefficients functions $\Delta C_{i}$ have a perturbative expansion

$$
\Delta C_{i}\left(x, \alpha_{s}\right)=\Delta C_{i}^{0}(x)+\frac{\alpha_{s}\left(Q^{2}\right)}{2 \pi} \Delta C_{i}^{(1)}(x)+\cdots
$$

The LO terms are simply

$$
\Delta C_{q}^{0}=\delta(1-x) \quad \Delta C_{g}^{0}=0
$$


and the NLO corrections are scheme dependent; typical choices differ in the amount of gluon contribution to the quark singlet distributions, while quark non-singlet distributions are scheme independent [1]. Finally, the $Q^{2}$ evolution of the parton densities obeys the DGLAP evolution equations [2], and, if known at an initial scale $\mu^{2}$, the r.h.s. of Eq. (1) can be computed at any perturbative $Q^{2}$ value.

By comparing data on $g_{1}\left(x, Q^{2}\right)$ with Eq. (1) one obtains information on the quark and gluon helicity distributions; the more data one has and the wider the $x$ and $Q^{2}$ range is, the more stringent the comparison is. The normal procedure is that of using a simple ansatz for the unknown distribution functions at the initial scale $\mu^{2}$, with some assumptions regarding the sea quark densities (for example, whether $S U(3)_{F}$ symmetric or not) and some constraints from $S U(3)_{F}$ hyperon decay sum rules on the first moments $\Delta q\left(1, Q^{2}\right) \equiv \int_{0}^{1} \Delta q\left(x, Q^{2}\right) d x$.

In Fig. 2 a most recent analysis of the world data on $x g_{1}(x)$ is shown together with a fit from Ref. [3], where the resulting helicity distributions can also be seen. Several similar analyses can be found in the literature; a complete list of references is given in Ref. [3].

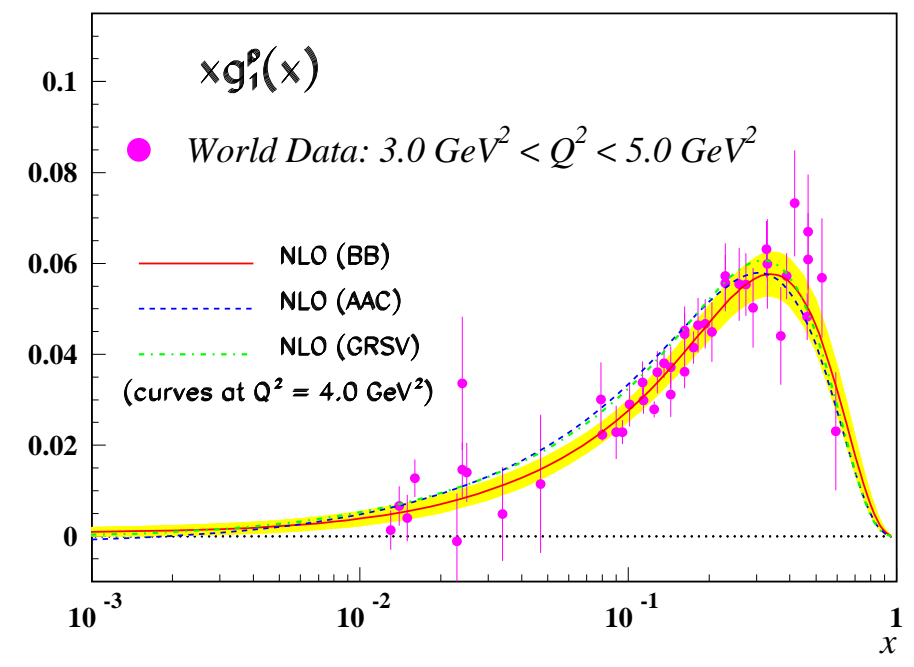

Figure 2: The world data on $x g_{1}^{p}(x)$; the figure is taken from Ref. [3]

Some data on $x g_{2}(x)$ are also available and the most recent ones [4] are shown in Fig. 3.

Let us shortly comment on these experimental results and the information which they offer.

- We have now good data on $g_{1}$ and $g_{2}$, although not yet comparable with the amount and quality of similar data obtained on the unpolarized structure functions.

- $g_{1}$ [see Eq. (1)] allows to obtain information on linear combinations of $(\Delta q+$ $\Delta \bar{q})$. We still need a better flavour resolution; this might come from semiinclusive DIS which gives information on $\sum_{q} \Delta q D_{q}^{h}$ rather than $\sum_{q} e_{q}^{2}(\Delta q+$ 


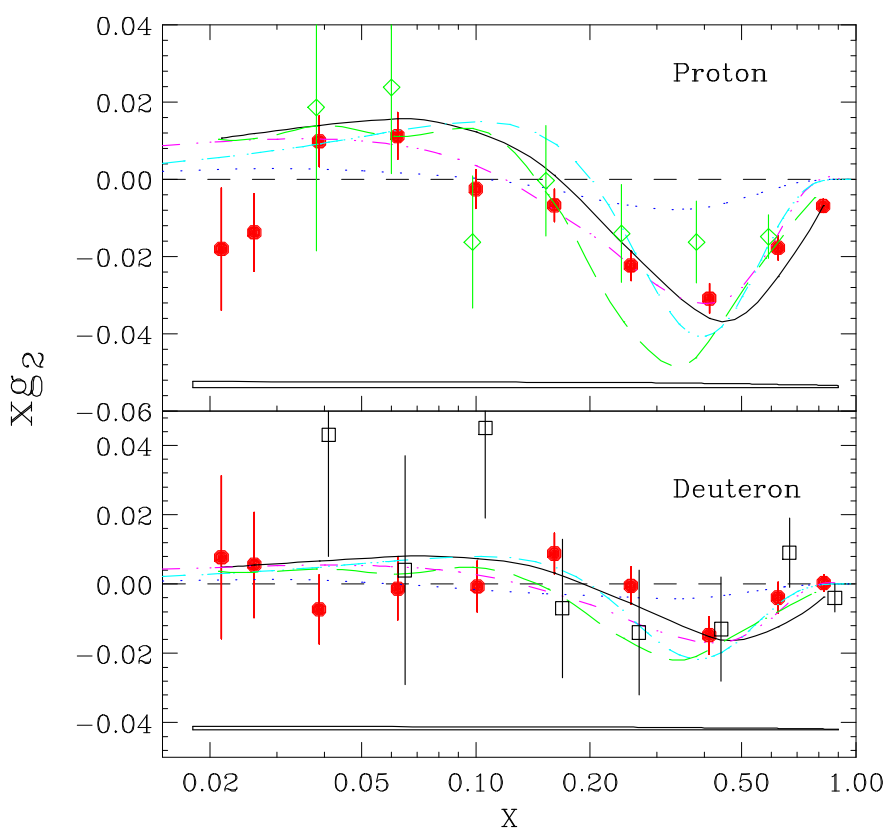

Figure 3: The E143 and E155 data on $x g_{2}^{p}(x)$ and $x g_{2}^{d}(x)$, from Ref. [4].

$\Delta \bar{q})$, where $D_{q}^{h}$ is the quark $q$ fragmentation function into the observed hadron $h$. Flavour separation in inclusive DIS would naturally be possible in neutrino iniziated charged current processes [5].

- Eq. (1) also offers indirect (via QCD evolution) information, on $\Delta g$. This is not stringent enough and a more direct measurement of the gluon helicity distribution is needed. This might come from the study of spin dependences in processes like $\ell p \rightarrow \ell+2$ jets, $\ell p \rightarrow \ell+c+\bar{c}+X, p N \rightarrow \gamma+X$, etc. which could be performed at HERMES, COMPASS, RHIC.

\section{Sum rules and orbital angular momentum}

In extracting information from experimental data - or in testing theories - a special role is plaid by sum rules. Let us mention a few of them.

The Bjorken sum rule $\left(g_{A} / g_{V}=1.2670 \pm 0.0035\right)$,

$$
\begin{aligned}
& \int_{0}^{1}\left[g_{1}^{p}\left(x, Q^{2}\right)-g_{1}^{n}\left(x, Q^{2}\right)\right] d x \\
= & \frac{1}{6} \frac{g_{A}}{g_{V}}\left\{1-\frac{\alpha_{s}}{\pi}-\frac{43}{12} \frac{\alpha_{s}^{2}}{\pi^{2}}-20.2 \frac{\alpha_{s}^{3}}{\pi^{3}}+\cdots\right\},
\end{aligned}
$$

is used in many ways. One can simply assume the validity of Eq. (5) and deduct from data on $g_{1}$ the value of $\alpha_{s}$ [6]; or one can use in it a value of $\alpha_{s}$ otherwise obtained, to check whether data on $g_{1}$ obey the sum rule or not (the answer is yes); or, also [7], one can assume to know the r.h.s. of Eq. (5), and see - among the poorly known behaviours of $g_{1}(x)$ at small $x$ - which one best satisfies the sum rule. 
Another, more debated, sum rule is the so called Burkhardt-Cottingham sum rule, according to which $\int_{0}^{1} g_{2}\left(x, Q^{2}\right) d x=0$, provided the integral exists. The recent E155 data [4] of Fig. 3 seem to indicate $\int_{0.02}^{0.8} g_{2}^{p}\left(x, Q^{2}\right) d x=-0.042 \pm 0.008$, which, taking into account uncertainties in the extrapolation to $x=0$ and $x=1$, might be the first indication of a violation of the sum rule, assuming that no $\delta$-function contributes at the origin [8].

The last, fundamental sum rule which we mention is the spin sum rule:

$$
\frac{1}{2}=\frac{1}{2} \Delta \Sigma(1)+\Delta g(1)+L_{q}+L_{g}
$$

where $\Delta \Sigma(1)$ is the first moment of $\sum_{q}[\Delta q(x)+\Delta \bar{q}(x)]$ and $L_{q, g}$ is the third component of the orbital angular momentum carried by quarks, gluons. This last quantity is unavoidable in a picture of the proton like that of Fig. 1: a spin 1/2 massless quark can emit a spin 1 massless gluon, via a helicity conserving coupling, only if some orbital angular momentum restores the total angular momentum conservation. However, there is little agreement at the moment both about the proper formal definition of a $\hat{L}_{q, g}$ operator and about a possible measurement of its expectation value between proton states [9].

\section{Transversity distribution}

The trasverse polarization of quarks inside a trasversely polarized nucleon, denoted by $h_{1}, \delta q$ or $\Delta_{T} q$, is a fundamental twist-2 quantity, as important as the unpolarized distributions $q$ and the helicity distributions $\Delta q$. It is given by

$$
h_{1}\left(x, Q^{2}\right)=q_{\uparrow}^{\uparrow}\left(x, Q^{2}\right)-q_{\downarrow}^{\uparrow}\left(x, Q^{2}\right),
$$

that is the difference between the number density of quarks with transverse spin parallel and antiparallel to the nucleon spin. It is the same as the helicity distribution only in a non relativistic approximation, but it is expected to differ from it for a relativistic nucleon.

When represented in the helicity basis (see Fig. 4) $h_{1}$ relates quarks with different helicities, revealing its chiral-odd nature. This is the reason why this important quantity has never been measured in DIS: the electromagnetic or QCD interactions are helicity conserving, there is no perturbative way of flipping helicities and $h_{1}$ decouples from inclusive DIS dynamics, as shown in Fig. 4a.

However, it can be accessed in semi-inclusive DIS, where some non perturbative chiral-odd effects may take place in the non perturbative fragmentation process, Fig. 4b. Indeed, a serious program to measure $h_{1}$ in semi-inclusive DIS at HERMES, where a transversely polarized proton target is now available, is in progress. A similar program, in different, complementary, kinematical regions, is planned at COMPASS.

The transversity distribution is also accessible at RHIC, where transversely polarized proton beams are available; by measuring double transverse spin asymmetries in Drell-Yan processes one obtains an observable which depends on the convolution 


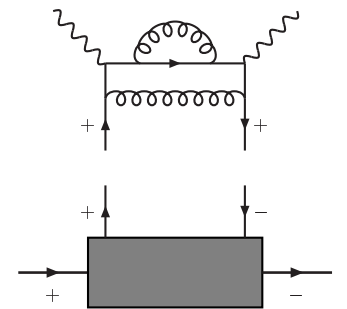

(a)

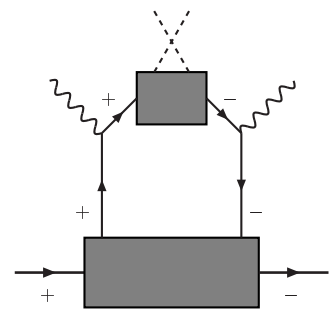

(b)

Figure 4: The chiral-odd function $h_{1}$ (lower box) cannot couple to inclusive DIS dynamics, even with QCD corrections; it couples to semi-inclusive DIS, where chiralodd non perturbative fragmentation functions may appear.

of two transversity distributions, which might make the overall effect rather tiny [10]. In general, $h_{1}$ must appear in a physical observable coupled to another chiral-odd quantity, which is either the transversity itself or a new unknown function.

\section{Unintegrated distribution and fragmentation functions}

We conclude by mentioning a new phenomenological approach to the description of many single transverse spin asymmetries which have been measured and keep being measured, with unexpected and interesting results [11]. The apparent problem with these asymmetries is related to the fact that, within perturbative QCD and the collinear factorization scheme, they should be vanishing, which is not true experimentally.

Recently, a series of papers [12] have shown how single spin asymmetries may occurr at the level of parton distributions and fragmentations, provided one takes into account the intrinsic motion of partons inside hadrons and of hadrons relatively to the fragmenting parton. For example, there might be a correlation between the transverse spin of a quark and the $\boldsymbol{k}_{\perp}$ of a resulting hadron, say a pion. This is the so-called Collins effect [13], pictorially shown in Fig. 5.

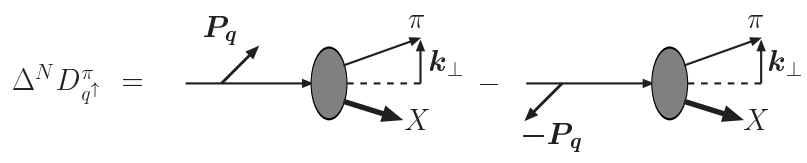

Figure 5: Pictorial representation of Collins function; notice that a similar function is sometimes denoted by $H_{1}^{\perp}$ in the literature.

Similar spin- $\boldsymbol{k}_{\perp}$ correlations may occurr also in the fragmentation of an unpolarized quark into a polarized hadron (the so-called polarizing fragmentation functions [14]), in the distribution of unpolarized quarks inside polarized nucleons (the Sivers effect [15]) and in the distribution of polarized quarks inside an unpolarized hadron $[16]$. 
When generalizing the factorization scheme with the inclusion of intrinsic $\boldsymbol{k}_{\perp}$, both in the distribution/fragmentation functions and in the elementary interactions, single transverse spin asymmetries appear immediately as possible and even sizeable. A phenomenological approach can be developed in which experimental information on the new functions is obtained from some processes and then used to make predictions in other cases.

The spin structure of the nucleon is subtle and challenging. Enormous progress has been achieved in the last years; yet, new surprising experimental results keep beeing obtained and fresh, interesting ideas keep being suggested. A lot more good work, both experimental and theoretical, is in progress.

Acknowledgments I would like to thank the organizers of the Symposium for the invitation and for the beautiful and stimulating organization.

\section{References}

[1] See, e.g., M. Anselmino A. Efremov and E. Leader, Phys. Rep.261, 1 (1995); B.W. Filippone and X. Ji, Advances in nuclear physics 26, 1 (2001), e-Print Archive: hep-ph/0101224.

[2] V.N. Gribov and L.N. Lipatov, Sov. J. Nucl. Phys. 15, 138 (1972); Y.L. Dokshitzer, Sov. Phys. JETP 16, 161 (1977); G. Altarelli and G. Parisi, Nucl. Phys. B126, 298 (1977).

[3] J. Blümlein and H. Böttcher, Nucl. Phys. B636, 225 (2002).

[4] E155 Collaboration (P. L. Anthony et al.), e-Print Archive: hep-ex/0204028.

[5] S. Forte, M. Mangano and G. Ridolfi, Nucl. Phys. B602, 585 (2001); M.L. Mangano et al., e-Print Archive: hep-ph/0105155.

[6] G. Altarelli, R.D. Ball, S. Forte and G. Ridolfi, Nucl. Phys. B496, 337 (1997).

[7] A. Knauf, M. Meyer-Hermann and G. Soff, Phys. Lett. B549, 109 (2002).

[8] M. Burkardt and Y. Koike, e-Print Archive: hep-ph/0206042.

[9] For a nice discussion see R.L. Jaffe, e-Print Archive: hep-ph/0102281; e-Print Archive: hep-ph/0101280.

[10] G. Bunce, N. Saito, J. Soffer and W. Vogelsang, Ann. Rev. Nucl. Part. Sci. 50, 525 (2000), e-Print Archive: hep-ph/0007218.

[11] For a review, see, for example, M. Anselmino, e-Print Archive: hep-ph/0201150. 
[12] S.J. Brodsky, D.S. Hwang and I. Schmidt, Phys. Lett. B530, 99 (2002) and Nucl. Phys. B642, 344 (2002); J.C. Collins, Phys. Lett. B536, 43 (2002); X. Ji and F. Yuan, Phys. Lett. B543, 66 (2002).

[13] J.C. Collins, Nucl. Phys. B396, 161 (1993).

[14] P.J. Mulders and R.D. Tangerman, Nucl. Phys. B461, 197 (1996); B484, 538 (1997) (E); M. Anselmino, D. Boer, U. D'Alesio and F. Murgia, Phys. Rev. D63, 054029 (2001).

[15] D. Sivers, Phys. Rev. D41, 83 (1990); D. Sivers, Phys. Rev. D43 261 (1991); M. Anselmino, M. Boglione and F. Murgia, Phys. Lett. B362, 164 (1995).

[16] D. Boer, Phys. Rev. D60, 014012 (1999). 